\title{
A Case Report: Diabetic Ketoacidosis in Patient with type 1 Diabetes Mellitus with Complication Septic Shock and AKI Stage III on HD Triggered by Perianal Abscess
}

\author{
Muhammad Ikhsan Kartawinata ${ }^{1 *}$, Yusni Puspita ${ }^{1}$ \\ ${ }^{1}$ Departement of Anesthesiology and Intensive Care, Faculty of Medicine, Universitas Sriwijaya, Palembang, \\ Indonesia
}

\author{
*Corresponding author: \\ Muhammad Ikhsan Kartawinata \\ Departement of Anesthesiology and \\ Intensive Care, Faculty of \\ Medicine, Universitas Sriwijaya, \\ Palembang, Indonesia \\ Email: \\ ikhsan_k@gmail.com
}

https://doi.org/10.37275/jacr.v1i1.135

\begin{abstract}
Introduction. Diabetic ketoacidosis (DKA) is an acute, life-threatening complication in diabetes mellitus. Infection is a common precipitating cause of diabetic ketoacidosis (DKA) in known diabetic patient, and diabetic ketoacidosis (DKA) often presents as the first symptom of an undiagnosed diabetes. diabetic ketoacidosis (DKA) is diagnosed with combination of hyperglicaemia, acidosis metabolic and ketonuria.

Case Presentation. A 27 years old male patient, admitted to Intensive Care Unit with decrease level of consciousness (GCS 3), he was intubated and present with respiratory distress, metabolic acidosis, high glucose level, ketonuria with renal failure as a target organ. Patient known has perianal abscess as a triggered of diabetic ketoacidosis (DKA), turn into septic shock and underwent debridement surgery to source control the infection. The patient was treated in intensive care unit for 9 days, and sent to ward with GCS 15 an no sequelae of organ failure. The treatment of diabetic ketoacidosis (DKA) should include correcting the often substantial hypovolemia, the hyperglycemia, electrolyte imbalance and the triggering factor of diabetic ketoacidosis (DKA). Conclusion. Prompt surgical intervention, antibacterial therapy, rapid restoration of glycemic control are crucial to prevent mortality in diabetes mellitus patients complicated with abscess.
\end{abstract}

Keywords. Diabetic Ketoacidosis, Septic Shock, Adult Respiratory Distress Syndrome, Ketosis, Hyperglycemia. 


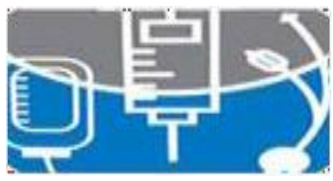

\section{Journal of Anesthesiofogy \& \\ Clinical Research}

\section{Introduction}

Diabetic ketoacidosis (DKA) is an acute, life-threatening complication in diabetes mellitus. Diabetic ketoacidosis (DKA) results from severeinsulin deficiency and mainly occurs in type 1 diabetes. Infection is a common precipitating cause of diabetic ketoacidosis (DKA) in known diabetic patient, and diabetic ketoacidosis (DKA) often presents as the first symptom of an undiagnosed diabetes. Vomiting, nausea, deep gasping breathing (kussmaul respiration), pronounced thirst, malaise and general weakness are typical symptom of diabetic ketoacidosis (DKA). Patients may also present with altered consciousness, disorientation, confusion or occasionally coma when the condition is severe. Diabetic ketoacidosis (DKA) is diagnosed with combination of hyperglicaemia, acidosis metabolic and ketonuria. The treatment of diabetic ketoacidosis (DKA) should include correcting the often substantial hypovolemia, the hyperglycemia, and electrolyte imbalance.

\section{Case Presentation}

A 27 years old male patient, admitted to Intensive Care Unit with decrease level of consciousness (GCS 3), he was intubated and present with respiratory distress with respiratory rate 44x/min, high glucose level (446 mg/dl), ketonuria and increase of ureum $(282 \mathrm{mg} / \mathrm{dl})$ and creatine level $(8,72 \mathrm{mg} / \mathrm{dl})$. The patient was found to have a metabolic acidosis with $\mathrm{PH}$ of 7.245, HCO3 6,8 mEq/L, pO2 $104 \mathrm{~mm} \mathrm{Hg}$ and pCO2 15,6 mmHg. Patient diagnosed with type 1 diabetes mellitus since 2 months ago and treated with combination long and rapid acting subcutaneous insulin. In ICU, patient known has perianal abses, and blood pressure was decrease $84 / 42 \mathrm{mmHg}$, heart rate 123 beats/min treated with norepinefrin $0,05-$ $0,5 \mathrm{mcq} / \mathrm{kgBB} / \mathrm{minand}$ underwent debridement surgery to source control the infection. Patient also did Haemodialysis to treat the acute kidney injury (AKI). The blood glucose successfully controlled on day 4 treatment in ICU and the level of conciuosness was increased. Patient was extubated after attainment of normal hemodynamic, glucose level and adequate power and consciousness. The Renal function back to normal and patient sent to ward after 9 days treatment in ICU with GCS 15 and discharge 3 days later. 


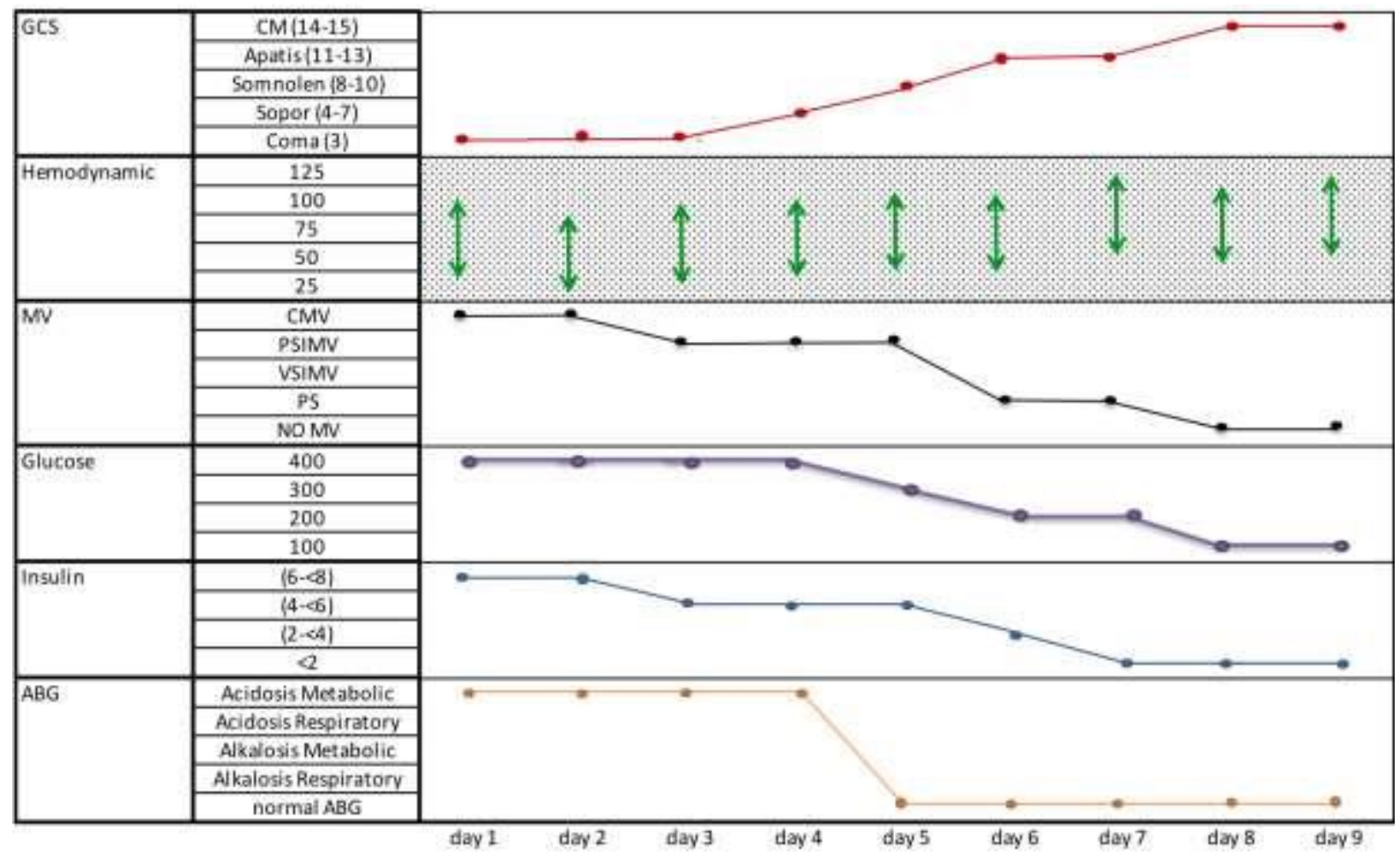

Figure 1. Patient progress during treatment in Intensive Care Unit. GCS, Glasgow Coma Scale; MV, Mechanical Ventilator; ABG, Arterial Blood Gas.

\section{Discussion}

Diabetic ketoacidosis (DKA) is a complication of decompensated diabetes mellitus. The sign of diabetic ketoacidosis (DKA) are primarily the result of abnormalities in carbohydrate and fat metabolism. Episodes of diabetic ketoacidosis (DKA) occur more commonly in patients with type 1 diabetes mellitus and are precipitated by infection or acute illness. Patients with diabetes mellitus are susceptible to infection because of decreased migratory ability of neutrophils, decreased phagocytic activity, impaired humoral immunity, increased adherence of microorganisms to diabetic cells, neuropathy and microangiopathy. Diabetic mellitus patients may be less able to defend against infection. Joshi et al, classified infection of the skin and soft tissue in diabetes mellitus patients into 2 types : mixed infection of Gram-negative bacilli and anaerob bacteria and infection of Gram-positive cocci. Furthermore, in patients with abscesses, diabetes mellitus-induced microangiopathy is believed to stimulate the growth of anaerobic bacteria because of the lack of oxygen in the peripheral tissue and decreased availability of antibacterial agent at the site of inflammation. 


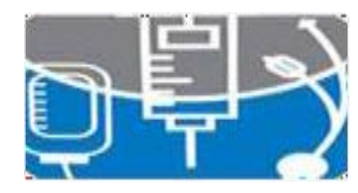

\section{Journal of Anesthesiology \& \\ Clinical Research}

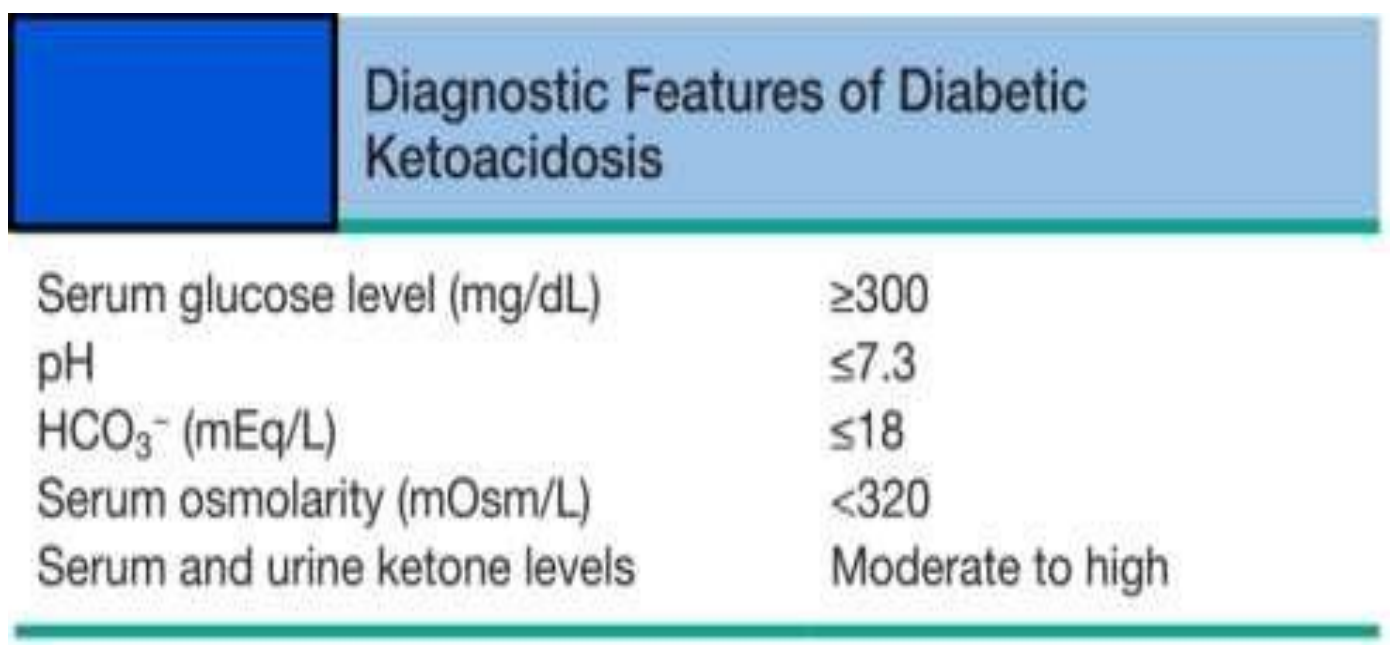

Figure 2. Diagnostic Features of Diabetic Ketoacidosis. (references: Stoelting's Anesthesia and Coexisting Disease. 7th Ed. Endocrine Disease)

In abscesses of the skin and soft tissue, early surgical treatment will improve prognosis in diabetes mellitus patients. In the present case, debridement were also perform early. Hyperglycaemia may be mild in the presence of a good renal function. But as the disease process evolves, glucose induced osmotic dieresis leads to volume depletion and secondary renal failure.

Treatment of diabetic ketoacidosis (DKA) consist of administration of large amounts of normal salin, effective doses of insulin, and electrolyte supplementation. The goal for decreasing blood glucose in keto-acidosis should be $75-100 \mathrm{mg} / \mathrm{dl} / \mathrm{h}$ or $10 \% / \mathrm{h}$. as glucose moves intracellularly, so does potassium . Although this can quickly lead to a critical level of hypokalemia if not corrected. Potassium and blood glucose should be monitored frequently during treatment of diabetic ketoacidosis (DKA). Several liters of $0.9 \%$ saline $(1-2 \mathrm{~L}$ the first hour, followed by $200-500 \mathrm{ml} / \mathrm{h}$ ) may be required to correct dehydration. Patients may benefit from precise monitoring of urinary output during initial treatment of diabetic ketoacidosis (DKA).

\section{Conclusions}

Early diagnosis and management of infection in diabetics with diabetic ketoacidosis (DKA) are mandatory. Although the mortality rate associated with diabetic ketoacidosis (DKA) is low, treatment of the triggering factor is essential component of the management of diabetic ketoacidosis (DKA). 


\section{Sournal of Anesthesiology 8 \\ Clinical Research}

\section{References}

1. Morgan GE, Mikhail MS, Murray MJ. Clinical Anesthesiology. 5th Ed. Anesthesia for Patients with Endocrine Disease. LANGE. 2013; 34: P 728-732.

2. Roberta LH, Katherine EM. Stoelting's Anesthesia and Co-existing Disease. 7th Ed. Endocrine Disease. ELSEVIER. 2018; 23;P 449-458.

3. Barash PG, Cullen FB, Stoelting RK. Clinical Anesthesia. 5th Ed, Lipincott Williams And Wilkins Company. 2006; 41: P 1145-49.

4. Kitabchi AE, Umpierrez GE, Murphy MB, Barrett EJ, Kreisberg RA, Malone JI, Wall BM. Management of hyperglycemic crises in patients with diabetes. Diabetes Care 2001;24: P 131153. 